\section{MEDIOS ALTERNATIVOS DE MEDELIIN [2]: Diagnóstico}

\author{
Mónica María Valle Flórez*
}

\section{RESUMEN}

En este segundo artículo sobre los medios alternativos de Medellín se presenta la caracterización de sus públicos así como algunos aspectos de su estructura organizativa. Se plantea que los medios alternativos conectan con los principios de la comunicación: expresión, conexión, imaginación ciudadana y entretenimiento.

El diagnóstico realizado se fundamentó teórica y metodológicamente desde un modelo de ciudad orientada al fortalecimiento de sus capacidades y las de sus ciudadanos, aspectos considerados por el entonces Alcalde de la ciudad Doctor Sergio Fajardo Valderrama, actual Gobernador de Antioquia, y la Caja de Compensación FamiliarComfama- entidad que ejecutó el proyecto.

El estudio partió de la premisa de que los medios alternativos podían constituirse en promotores de la convivencia ciudadana y que para ello sería conveniente realizar inventario y diagnostico de éstos, brindarles capacitación y estructurar la red de medios alternativos de Medellín.

\section{Palabras clave:}

Medios alternativos, comunicación ciudadana, red de medios alternativos, comunicación pública, convivencia ciudadana, Medellín.

\section{ALTERNATIUE MEDIA OF MEDELLÍN [2]: Diagnostic}

\author{
Mónica María Valle Flórez*
}

\section{SUMARY}

This second article about alternative media of Medellín presents the characterization of their audiences and some aspects of its organizational structure. It propound that alternative media connect with the communication principles: expression, connection, civic imagination and entertainment.

The diagnosis made, was based theoretically and methodologically from a city model oriented at strengthening of its capabilities and those of its citizens, these aspects were considered by the mayor of the time Doctor Sergio Fajardo Valderrama, who is the current Governor of Antioquia, and the Compensation Familiar Found Comfama (institution that implemented the project).

The study started off with the premise that alternative media could become promoters of civic coexistence and for this would be appropriate perform stocktaking and diagnostic of these, offer to them training and structure the network of alternative media of Medellin.

\section{Key words:}

Alternative media, civic communication, network of alternative media, public communication, civic coexistence, Medellín.

\footnotetext{
* Doctora en Estudios Científico Sociales- Instituto Tecnológico de Occidente - ITESO (México), Magister en Comunicación de la Universidad Iberoamericana de México, Especialista en Gerencia de la Comunicación de la Universidad Pontifica Bolivariana (Colombia) y Comunicadora Social Periodista de la Universidad de Antioquia (Colombia). Docente/ investigadora - Politécnico Colombiano Jaime Isaza Cadavid. Email: mmvalle@elpoli. edu.co
}

*PHD of Social Science Studies - West Institute of Technology-ITESO (Mexico), Master in Communication from the Universidad Iberoamericana of Mexico, Management Specialist Communication at Universidad Pontificia Bolivariana (Colombia), Social Communicator and Journalist of Universidad de Antioquia (Colombia). Teacher/ researcher - Politécnico Colombiano Jaime Isaza Cadavid. E-mail: mmvalle@elpoli.edu.co 


\section{Expresión, conexión, imaginación}

\section{ciudadana y entretenimiento}

Rincón (2005) plantea que la comunicación es importante por los valores, por los contenidos, por lo educativo, por lo formativo, por lo informativo, por la construcción de sociedad, por la negociación y por las estéticas. Indica que la expresión, la conexión, la imaginación ciudadana y el entretenimiento son acciones de la comunicación. Para este investigador la expresión es la primera acción dela comunicación. La segunda la conexión, al respeto indica:

"...se hacen medios comunitarios para conectar alrededor de unos discursos, de unos imaginarios, de unas formas de ser..."

La tercera acción es la imaginación ciudadana, esto es que los medios de comunicación deben ayudar a imaginar que hay una sociedad distinta desde lo ciudadano y desde los accesos públicos, por lo que:

"...los medios locales deben servir a construir relatos, historia, a construir ciudadanía comunicativa". Rincón (2005)

En cuarto lugar la comunicación debe entretener, al respecto argumenta Rincón:

"...nadie se sienta a verTV. para aburrirse, nadie prende el radio sólo por prenderla, nadie lee un periódico para decir: "como soy de culto". Uno entra porque quiere pasar un buen tiempo leyendo un periódico. El entretenimiento no quiere decir hacer reír. Entretenimiento quiere decir hacer pensar, hacer sentir, emocionar, producir algo en la audiencia. Entretenimiento es que me produzca algún efecto emocional como lugar de la comunicación".

El acelerado desarrollo tecnológico ha convertido a los medios en creadores de referentes culturales, comportamientos, modos de ser y hacer que luego las audiencias reproducen en sus espacios cotidianos.

Así como a los medios de comunicación social se les ha atribuido responsabilidad en los comportamientos agresivos de las personas, ese mismo poder de influencia, y su gran capacidad de penetración, deben ser aprovechados para favorecerygenerarunambientedeentendimiento y convivencia. Como indica Rincón (2005) los medios no se inventan los hechos de violencia, pero esa realidad que informan es un hecho que alguien percibe de cierta manera.

Los medios de comunicación deben decantar las realidades, en otras palabras, analizarle de manera compleja, de igual manera deben revisar las fuentes de donde se obtiene información sin caer en los viejos vicios de: guerra por las audiencias, reacción en el cubrimiento noticioso, prevalencia de los interés privados frente a los público, síndrome de la chiva, pérdida del rigor periodístico porelafán de contaralgo nuevo, invocarla"opinión pública" para evadir su responsabilidad política y moral, disputa desbocada por la sintonía, noticias descontextualizadas, periodismo de escritorio y registro de hechos sin beneficio de inventario, sin profundizar en sus orígenes y consecuencias.

\section{Intervención}

En Colombia se registra como antecedentes de la intervención a medios alternativos el realizado por la Universidad de Antioquia en el marco del Plan de Desarrollo del Departamento (2005) [1] y el "Encuentro Distrital de Comunicación Comunitaria" [ 2], evento convocado, ese mismo año, por la Alcaldía Mayor de Bogotá D.C.

En el realizado por la Universidad de Antioquia se encontró que la región contaba con 308 medios, divididos en 134 emisoras, 120 canales de televisión y 54 impresos. La distribución de los medios por subregiones se presentó así: 20 medios en Urabá, 18 en el Bajo Cauca, 20 en el Nordeste, 28 en el Norte, 27 en el Occidente, 17 en el Magdalena Medio, 53 en el Valle de Aburrá, 80 En el Oriente, y 45 en el Suroeste.

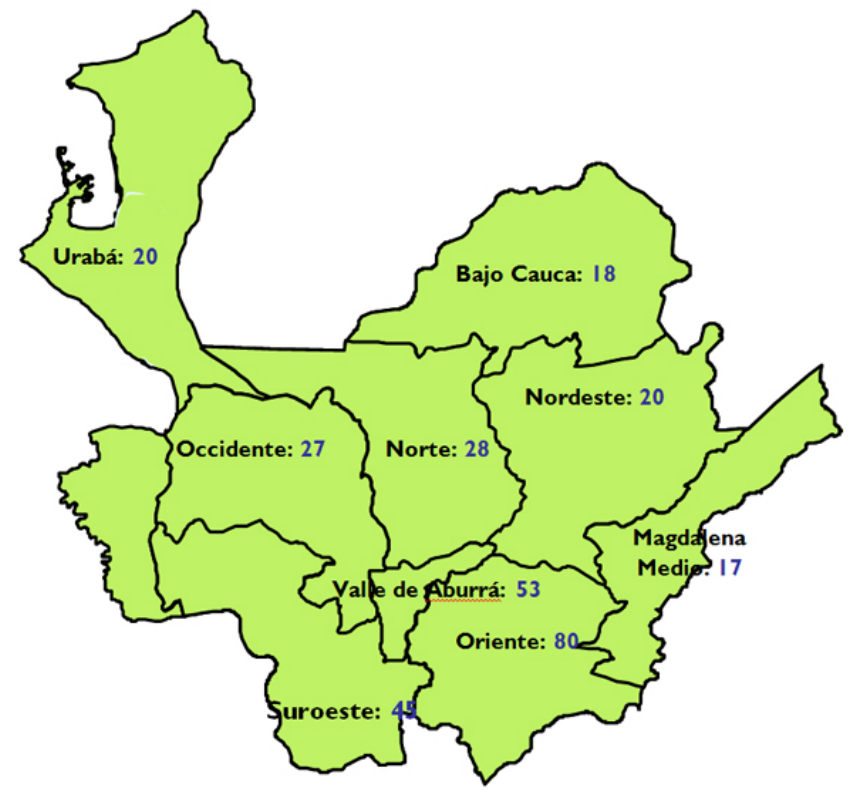

Fuente: Base de datos Comunicación, Vida y Territorio actualizada -2005 .

El "Encuentro Distrital de Comunicación Comunitaria" convocado por la Alcaldía Mayor de Bogotá D.C. tuvo como fin promover la democracia participativa y el fortalecimiento de la gobernabilidad local así como abrir un primer espacio de reflexión en torno a la necesidad de construir una política de comunicación comunitaria en Bogotá. Los ejes sobre los que giró esta propuesta fueron reconciliación y gestión pública humana. 
Con estos antecedentes se dio inició al

"Diagnóstico de los medios alternativos y comunitarios de Medellín"

cuyo objetivo fue estructurar una red de comunicación orientada a la convivencia ciudadana en Medellín y sus corregimientos. La consultoría comprendió fases de diagnóstico, cualificación de los integrantes de los medios alternativos, concurso de méritos, estructuración y acompañamiento para la red de medios constituida.

\section{Diagnóstico de Medios Alternativos en Medellín}

Desde finales de los 90 una serie de organizaciones sociales se dieron a la labor de impulsar medios alternativos en la ciudad de Medellín mediante talleres y capacitaciones.

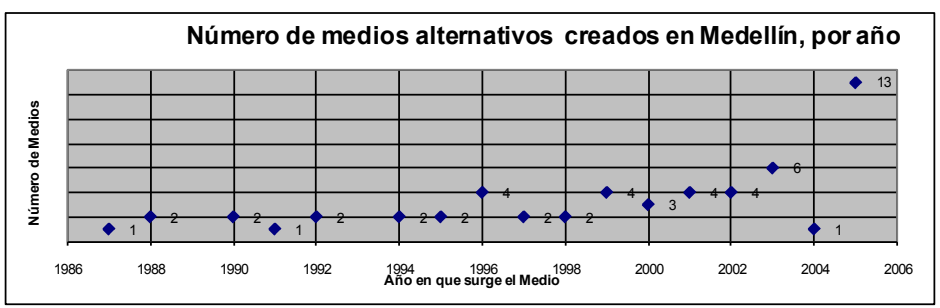

En este diagnóstico se encontró que en el 2003 se crearon seis nuevos medios, y que en el 2005 se presentaban 13 nuevas iniciativas, en su mayoría apoyadas con presupuesto participativo:

\begin{abstract}
“...con recursos del Programa de Planeación y Presupuesto Participativo varias organizaciones de las comunas y corregimientos de Medellín reforzaron sus medios de comunicación; crearon nuevos $\mathrm{o}$ adquirieron herramientas para entender la comunicación como un mecanismo importante de trabajo al interior de sus zonas. La Dirección de Comunicaciones de la Alcaldía de Medellín acompañó durante el 2005 todos estos procesos y asignó un comunicador para apoyar la definición de los distintos medios informativos así como la realización de talleres y actividades de capacitaciones en los temas de comunicación" [ 3]
\end{abstract}

Según informe de la Alcaldía Municipal de Medellín, el recurso asignado para estos proyectos fue de 519 millones [4]. A la fecha del estudio se determinó que la permanencia promedio de los medios era de tres años. Entre los factores de su desaparición se encontró: falta de fuentes de financiamiento, ocupación de los líderes de los medios en otros proyectos que les resultaban más estables o consolidados.

\section{Púlblico de los medios aliternativos}

La mayoría de los medios (35) establecieron que su público objetivo era la comunidad, sin embargo se encontraron medios cuyos públicos específicos eran: jóvenes (9), feligreses (4), corregimientos (2), niños (2), afrocolombianos (1), comerciantes (1), docentes (1), instituciones educativas (1).
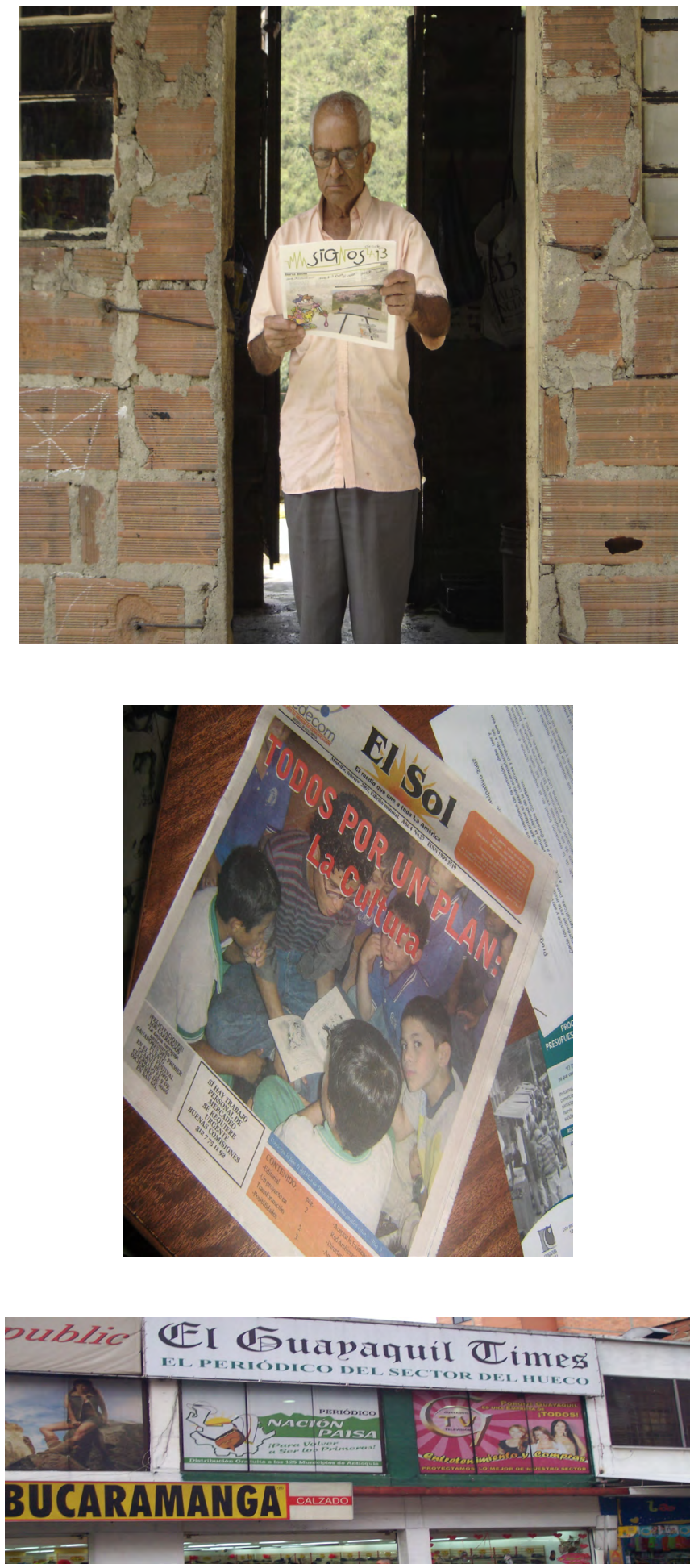

En relación a la ocupación de los perceptores, se encontró que en su mayoría (31\%) era vendedores, comerciantes y administradores, seguidos de amas de casa (23\%), empleados (20\%) y estudiantes (8\%). La distribución por género de los perceptores de los medios estudiados fue: masculino $59 \%$ y femenino $41 \%$. En cuanto a la edad el $51 \%$ eran adultos, el $33 \%$ adultos mayores, el $14 \%$ jóvenes y el $2 \%$ niños. 

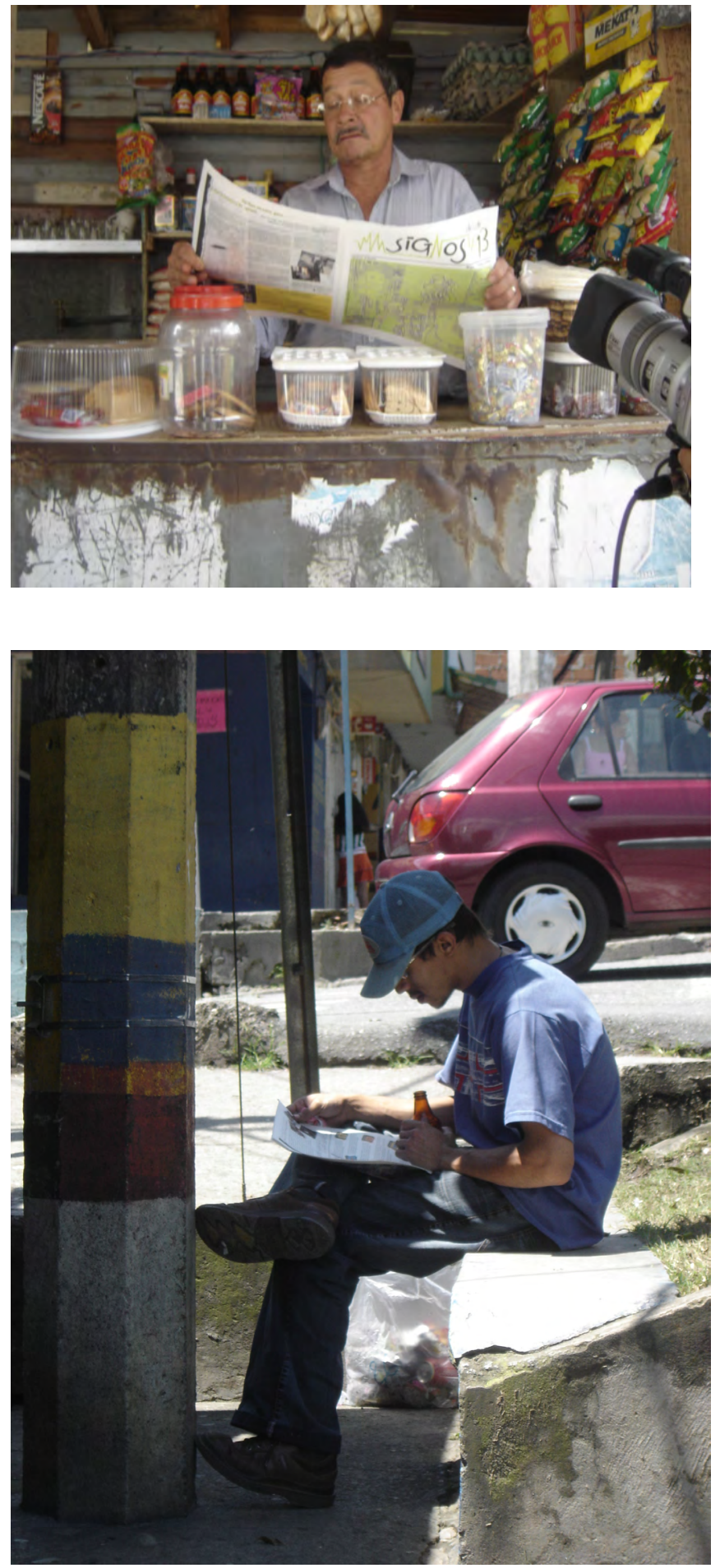

Fotos: Natalia Isaza y Laura Palacio

Los perceptores calificaron con 4 y 5 su credibilidad, sentido de pertenencia y el manejo informativo de los medios alternativos. El factor determinante de la buena valoración fue la cercanía de los integrantes del medio con la comunidad.

\section{Sistema Organizativo}

En el 2006, los medios alternativos de Medellín se constituían en una fuente de ocupación, no necesariamente remunerada, para 224 personas. En total, en los medios escritos se ocupaban 54 personas, en TV 113, en radio 40 y en medios Web 17.

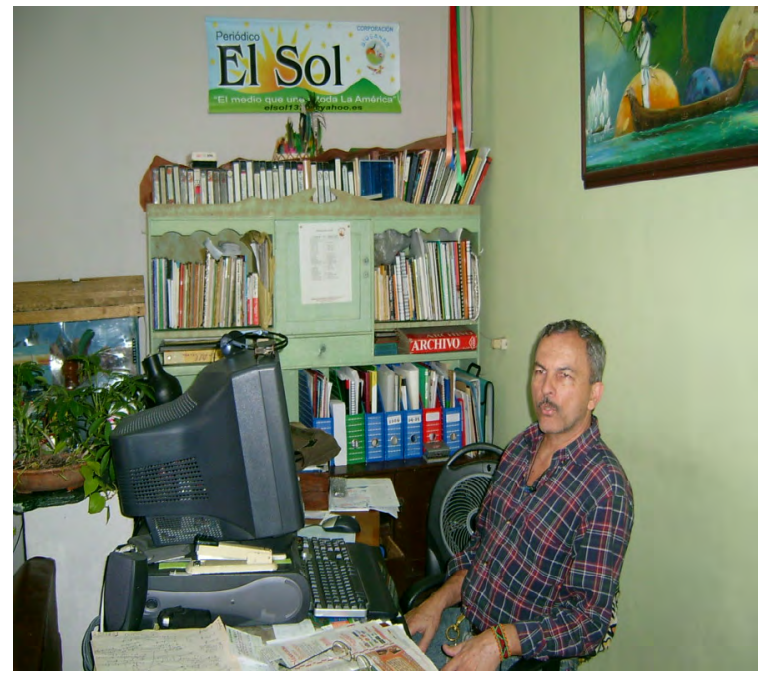

Hernán Atehortua- Director Periódico El Sol Foto: Juliana Díaz Cardona y Diana Lambis Murcia

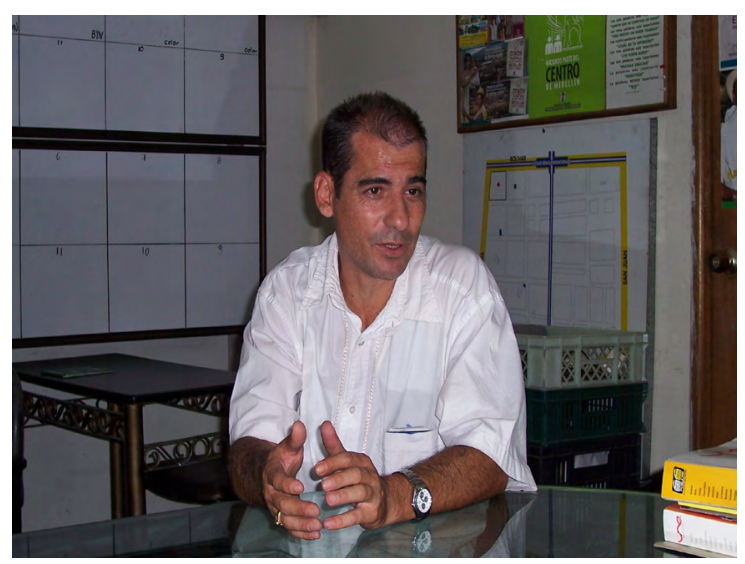

Jairo Bernal- Director Periódico El Guayaquil Times Foto: Natalia Andrea Díaz Mesa

La edad promedio del personal dedicado a labores en los medios alternativos de Medellín, a la fecha de este estudio, era de 30 años (50\%); 40 años (22\%), 19 años (13\%), más de 50 años (15\%) éstos últimos en su mayoría, jubilados. En relación con el nivel educativo se encontró que el $36 \%$ eran bachilleres, el 30\% contaban con estudios primarios, el $20 \%$ con estudios universitarios y el $13 \%$ con estudios técnicos.
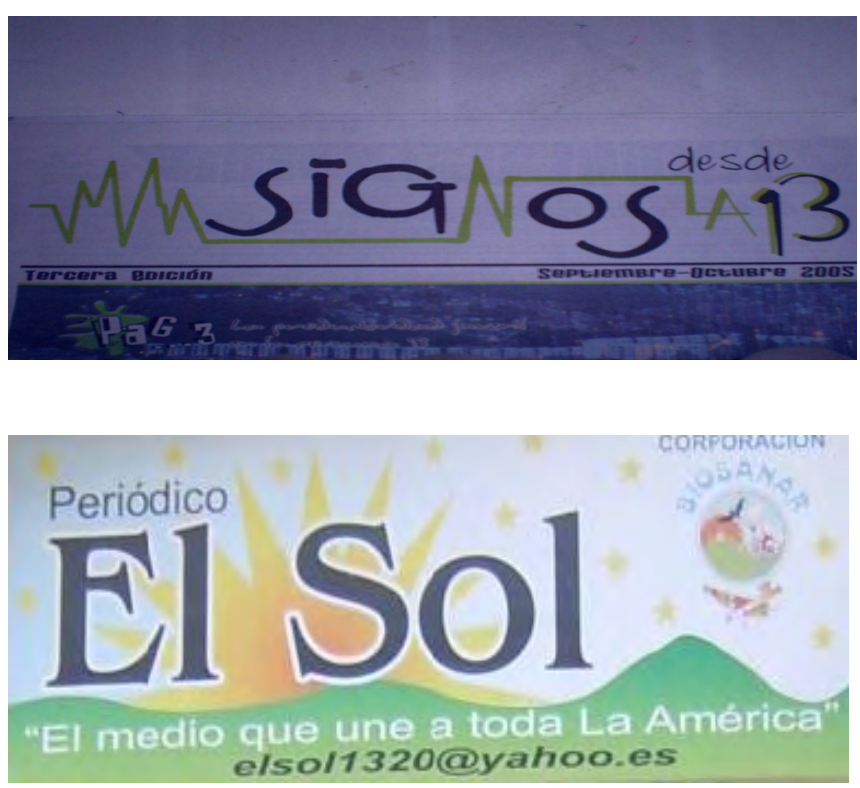


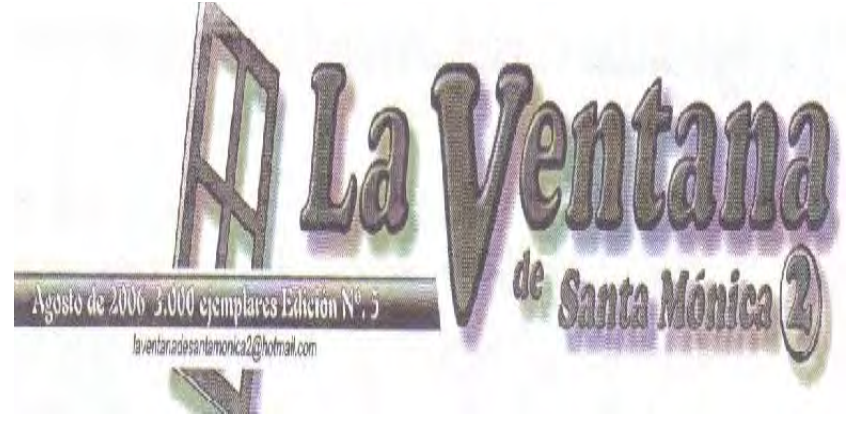

En cuanto a las profesiones, se determinó que el personal que se ocupaba en los medios alternativos de Medellín (2007), en su mayoría, eran comunicadores, publicistas, diseñadores gráficos, fotógrafos, locutores, docentes, actores, cuenteros, sacerdotes, filósofos, sociólogos, antropólogos y administradores. También ingenieros de sistemas (2), un médico y una odontóloga.
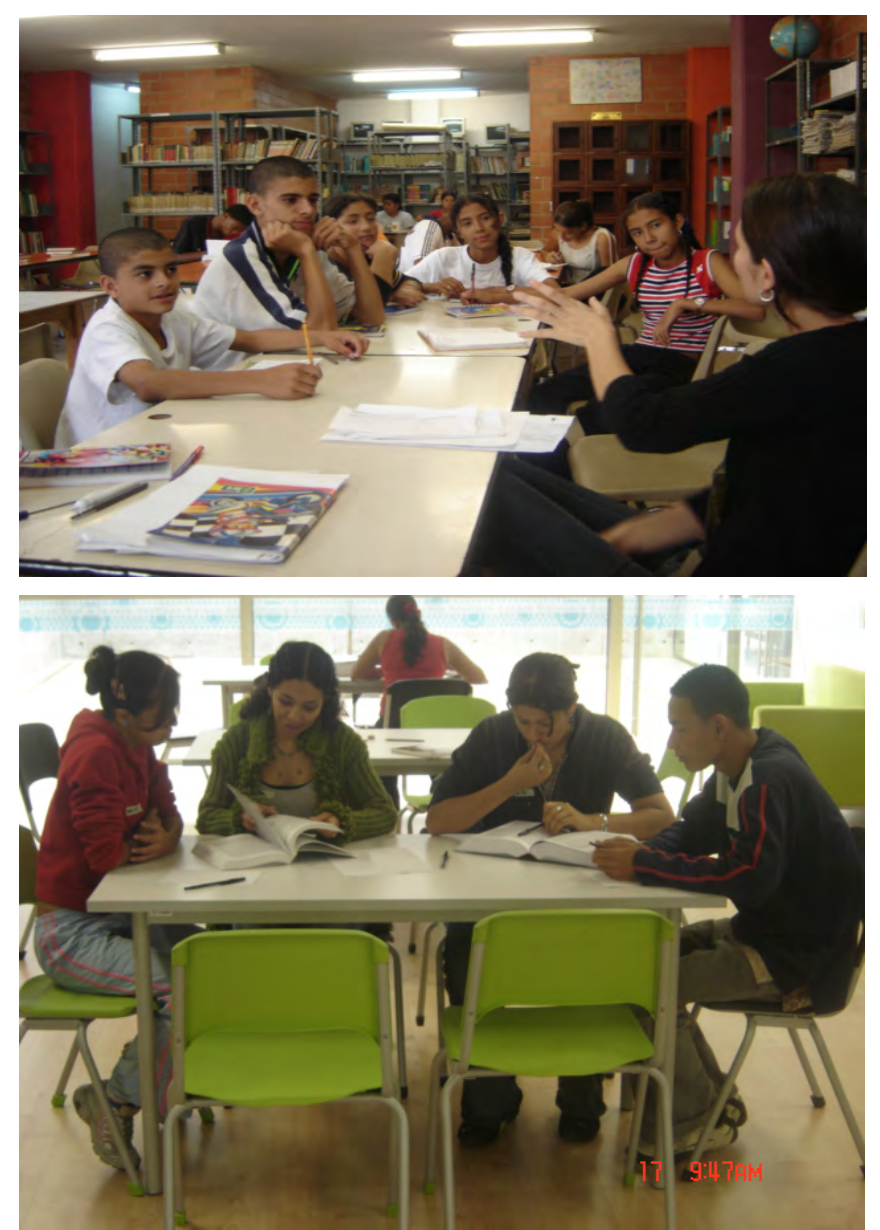

Fotos: Natalia Isaza y Laura Palacio

En la mayoría de los medios consultados se encontró que contaban con cargos definidos y distribución de trabajo. Sus estructuras organizativas, en general, se catalogaron como medianamente desarrolladas. Los medios escritos y de TV presentan mayor desarrollo organizativo.

\section{Entre los cargos establecidos, en cada medio alternativo, se encontraron:}

- Medios Escritos: Director, subdirector, gerente, periodista, colaboradores, promotores, diseñadora gráfica, vendedor, consejo de redacción, secretaria, mensajero.

- En televisión se encontraron los cargos de productores, técnicos y auxiliares de oficina, gerencia, camarógrafo, editor, periodista, jefe de producción, investigador, control master, sonidista, luminotécnico, coordinador de piso y asesores.

- La radio y la Web presentan estructuras organizativas incipientes; en el primero se cuenta con los cargos de coordinador de emisora, reporteros y locutores, en el segundo con Webmaster.

Se establecieron como las principales fuentes de financiación de estos medios, la pauta publicitaria, los aportes de la comunidad, recursos del presupuesto participativo y recursos propios, la venta directa del medio, apoyo de la Administración Municipal y las donaciones.

\section{Red de Medios Alternativos de Medellín}

La estructuración de la Red de Medios Alternativos de Medellín contó con el aval de los convocados. A la fecha de conclusión de este diagnóstico (2007), se había conformado la mesa de trabajo. Las ventajas que le concedieron a la red fueron:

\begin{abstract}
"Autogestión solidaria como principio económico sostenible del medio de comunicación"; "posibilidad de impulsar una red de televidentes comunitarios"; "intercambiar programas, artículos, experiencias así como mejorar la calidad de los medios", etc.
\end{abstract}

\section{Se estableció que la Red contribuía a:}

\begin{abstract}
"Generar arraigo e identidad local"; "compartir base de datos entre los medios"; "crear, integrar e impulsar proyectos sociales y económicos que involucraran a los integrantes de la Red"; "Crear una escuela de capacitación en comunicación con acceso para todos", etc.
\end{abstract}

\section{Algunos de los principios adoptados para el trabajo en la red fueron:}

- Facilitar la libertad y la autonomía

- Tolerancia: conocer y respetar la diferencia

- Equidad

- Oportunidad: atención inmediata a la necesidad expresada por la comunidad con una respuesta humanizada, integral, interinstitucional y eficiente

- Solidaridad: apoyo mutuo y trabajo colectivo.

- Reconocimiento del trabajo de las diferentes organizaciones. 


\section{Señala (Quiroz, 2007) que como sistema organizativo una red debe:}

- Definir un marco conceptual.

- Diagnosticar el Problema: visión de las instituciones sobre éste.

- Contar con principios filosóficos.

- Definir objetivos y metas: deben responder a las necesidades reales de la comunidad e instituciones.

- Definir metodología de trabajo

- Determinar acciones concretas para el logro del objetivo.

- Gestionar recursos: aportes de cada una de las instituciones a la red.

- Contar con mecanismos de evaluación y control que permitan dar cuenta de las acciones y logros.

\section{Los miembros fundadores de la Red se propusieron:}

- Contribuir al cumplimiento, desarrollo de políticas, planes y programas de los medios y organizaciones.

- Desarrollar metodologías que estimularan el trabajo interinstitucional, interdisciplinario, intersectorial y comunitario estableciendo mecanismos de concertación, coordinación y toma de decisiones.

- Fomentar el desarrollo metodológico y técnico de los representantes en torno a problemáticas que aborda la Red.

- Promover y fortalecer los medios e instituciones que le integran.

- Establecer sistemas de monitoreo, evaluación y control que permitieran observar el desarrollo de los planes y programas propuestos por la Red

- Permitir la ampliación de coberturas en términos de perceptores y participación activa de las comunidades.

- Promover la sensibilización, definición y utilización de programas y campañas dirigidos a la convivencia ciudadana.

- Comprender la heterogeneidad de las regiones en sus aspectos históricos, geográficos, culturales, económicos y sociales que puedan afectar la coordinación interinstitucional y comunitaria para dar respuesta a un problema común.

\section{Dificultades del trabajo en red}

Algunas de las dificultades de trabajo en red se fundamentan esencialmente en las mismas que impiden la construcción de tejido social y redes de ayuda. Algunas de estas son:
- Desconfianza entre los miembros de la red.

- Ausencia de claridad con respecto a los beneficios que produce la asociación.

- Percepción o temor por parte de los miembros con respecto al "robo o copia de metodologías" entre los medios asociados.

- Percepción o temor por parte de los miembros con respecto a que la red pueda suplantar el trabajo de las entidades socias.

- Temores de evidenciar públicamente déficit institucional.

- Prevención para compartir información de interés mutuo.

- Prevención para compartir fuentes de información comunes a estos medios

- Temores a que los financiadores puedan interesarse por otras instituciones de la red.

- Baja calidad de las funciones ofrecidas por el nodo de la Red y ausencia de mecanismos de estímulo para permanecer.

- Inmadurez de las entidades miembros.

- Visiones excesivamente diferentes.

- Saturación de redes y asociaciones de medios.

\section{Se contempló que Red de Medios Alternativos de Medellín tendría entres sus funciones:}

- Ser elemento mediador de intercambios simbólicos que aportara insumos útiles a la construcción de nuevos conocimientos.

- Ser mediadora cultural.

- Contextualizar la información.

- Establecer diferencias estratégicas en relación con otros medios.

- Gestionar Relaciones Públicas que le permitieran a los medios identificar políticas y procedimientos que posibilitaran adelantar programas de acción para conseguir la aceptación y el entendimiento de los diferentes públicos.

- Contribuir a la gestión organizativa de los medios.

\section{Conclusiones}

Los medios alternativos de Medellín son espacios de socialización de diferentes actores e ideologías, son espacios propicios para narrativas y estéticas diferentes a la de los medios masivos. "En el proceso de cambio de imaginarios los medios pueden ayudar a inventar otro futuro, ver otra realidad". 
El diagnóstico realizado evidencia que los medios alternativos de Medellín (2007) más que representados buscan ser reconocidos en sus diferencias.

Entre los antecedentes de intervención a medios alternativosseencontróelProyectoComunicación, Vida y Territorio- CONVITE liderado por el IDEA, la $U$ de $A$ y Comfenalco en el 2006. También se registró el Proyecto de la Administración Distrital de Bogotá (2005) encaminado a la construcción de una política de comunicación comunitaria en el Distrito Capital.

Este estudio recomendó establecer puntos de encuentro entre los resultados del proyecto Convite y otros realizados en la región con miras a impulsar un "cluster" de medios alternativos de Antioquia.

Incluir a los medios alternativos de Medellín y sus corregimientos, dentro de las estrategias para la convivencia ciudadana, implica un trabajo arduo de reflexión y acción conjunta con la comunidad y de igual manera dimensionar la comunicación pública como herramienta para la construcción democrática de sociedad.

La Red de Medios Alternativos de Medellín se pensó como estrategia para promover redes de conversación, intercambios de mundos simbólicos y expresivos que ayudaran a construir un nosotros de ciudad.

El estudio concluyó que en Medellín se debía impulsar y apoyar los medios alternativos en las comunas más desprovistas de estos: $2,3,6,8$ y 13 y en los corregimientos $50,60,70,90$. También que debía fomentar medios alternativos dirigidos a niños, jóvenes e impulsar una política estatal para los medios alternativos.

El mayor número de medios de la ciudad corresponde a medios escritos $51 \%$, le siguen los radiales $20 \%$, televisivos $16 \%$ y Web (13\%).

\section{Bībliografía}

Alcaldía de Medellín, Secretaria de Gobierno Municipal- Unidad de Convivencia Ciudadana. Boletín mensual Internet. 2005.

Conferencia Omar Rincón (2005). “Televisión pública y medios locales" Proyecto Convite.

Diagnóstico de los Medios Alternativos y Comunitarios de Medellín (2005-2006). Alcaldía de Medellín- COMFAMA.

Encuentro Distrital de Comunicación Comunitaria. Enrédate en Procesos de Comunicación. 12 y 13 de Diciembre- 2005. Bogotá. Colombia.

Propuesta Técnica de COMFAMA, para la Consultoría Estructuración de una Red de Medios de Comunicación orientada a la Convivencia Ciudadana de Medellín y sus Corregimientos. 2005.

Quiroz Margarita Inés, Memorias Violencia Intrafamiliar.

Valle Flórez, Mónica María (2012). MEDIOS ALTERNATIVOS DE MEDELLIN [1]: Recuento (19872006). Revista Virtual Luciérnaga, Año 4, N7. Grupo de Investigación en Comunicación, Facultad de Comunicación Audiovisual, Politécnico Colombiano Jaime Isaza Cadavid. MedellínColombia. ISSN 2017-1557. Págs. 36-46.

\section{Notas}

[1] Universidad de Antioquia, (2005). "Proyecto para el fortalecimiento de los medios locales de comunicación por la equidad y la vida en Antioquia" 2005.

[2] Encuentro Distrital de Comunicación Comunitaria (2005). Alcaldía Mayor de Bogotá D.C. 12 y 13 de diciembre. Auditorio Alfonso López Pumarejo- Universidad Nacional. BogotaColombia.

[3] Boletín 1398- Comunicaciones Alcaldía de Medellín- Diciembre 28-2005.

[4] Idem

\section{Para cìtar este artículo:}

Valle Flórez, Mónica María (2012). MEDIOS ALTERNATIVOS DE MEDELLIN [2]: Diagnóstico 2005-2006. Revista Virtual Luciérnaga, Año 4, N8. Grupo de Investigación en Comunicación, Facultad de Comunicación Audiovisual, Politécnico Colombiano Jaime Isaza Cadavid. Medellín- Colombia. ISSN 2027-1557. Págs. 51-57.

Diagnóstico de los Medios Alternativos y Comunitarios de Medellín (2005-2006). Alcaldía de Medellín- COMFAMA. Valle Flórez MónicaInvestigadora.

DOI. 10.33571/revistaluciernaga.v4n8a3 